\title{
Atención al paciente inmigrante: realidad y percepción de los profesionales de 6 zonas de Atención Primaria de Navarra
}

\section{Care for immigrant patients: facts and professionals' perception in 6 primary health care zones in Navarre}

\author{
M.C. Fuertes ${ }^{1}$, L. Elizalde ${ }^{2}$, M.R. de Andrés ${ }^{3}$, P. García Castellano ${ }^{4}$, S. Urmeneta ${ }^{5}$, \\ J.M. Uribe ${ }^{6}$, P. Bustince ${ }^{7}$
}

\section{RESUMEN}

Fundamento. Describir la utilización de servicios y motivos de consulta en Atención Primaria entre población autóctona e inmigrante y contrastarla con la percepción que tienen los profesionales de atención primaria.

Material y métodos. Se recogieron datos de actividad asistencial durante el año 2006 de la totalidad de personas adscritas $(\mathrm{N}=86.966)$ a las 6 zonas básicas con mayor proporción de población inmigrante (14,4\%) y las variables: país de origen, edad, sexo, año de alta en el sistema sanitario. Se utilizaron bases datos de tarjeta sanitaria y de programa OMI-AP. Se utilizó metodología cualitativa de grupos de discusión y entrevistas en profundidad.

Resultados. El 72,4\% de inmigrantes solicitaron atención de profesionales de atención primaria en 2006, de los cuales un 50\% procedía de Ecuador y un $70 \%$ tenía entre 25 y 44 años. Los autóctonos consultantes fueron el $82 \%$ y requirieron más derivaciones a atención especializada que los inmigrantes del mismo grupo de edad. La consulta más frecuente en autóctonos e inmigrantes fue "infecciones respiratorias agudas" (7 al 23\%, según grupos de edad). La segunda en inmigrantes fue "problemas administrativos". Las consultas de inmigrantes no se relacionan con aspectos preventivos como tabaquismo y sí tuvieron más consultas $(\mathrm{p}>0,001)$ de episodios gineco-obstétricos $(10,7 \%)$ y de los relacionados con problemas laborales (19\%) o psicosomáticos (8,5\%). La percepción de los profesionales de atención primaria era que la población inmigrante consulta más que la autóctona y genera cierto "desorden" en la consulta.

Conclusión. Los inmigrantes son menos utilizadores de servicios sanitarios y frecuentadores que los autóctonos. Sin embargo, este hecho no es percibido así por los profesionales de atención primaria. Con inmigrantes se realizan menos actividades preventivas y padecen más problemas laborales y psicosomáticos.

Palabras clave. Inmigrantes. Morbilidad. Centros de salud. Atención primaria de salud.

\begin{abstract}
Background. To describe utilisation of health care services and motives for consultation in Primary Care in the native and the immigrant population, and compare this with the perception of primary care professionals.

Methods. Data was collected on health care activity during the year 2006 for all people registered $(\mathrm{N}=86,966)$ in the 6 basic health care zones with the highest proportion of immigrants $(14.4 \%)$ and on the following variables: country of origin, age, sex, year of inscription in the public health service. The health card and OMI-AP programme databases were used. A qualitative methodology of focus groups and in-depth interviews was employed.

Results. Seventy-two point four percent of immigrants requested care from the primary care professionals in 2006 , of whom $50 \%$ proceeded from Ecuador and $70 \%$ were between 25 and 44 years old. Eighty-two percent of the natives made consultations and required more referrals to specialised care than the immigrants of the same age group. The most frequent consultation with natives and with immigrants was "acute respiratory infections" ( 7 to $23 \%$ according to age group). The second most frequent with immigrants was "administrative problems". The consultations with immigrants were not related to preventive aspects such as smoking and there were more consultations $(\mathrm{p}>0.001)$ for gynaeco-obstetric episodes $(10.7 \%)$ and those related to work (19\%) or psychosomatic problems $(8.5 \%)$ The perception of the primary care professionals was that the immigrants carry out more consultations than the natives and generate a certain "disorder" in the clinic.
\end{abstract}

Conclusion. Immigrants use healthcare services less than the native population. Nonetheless, this fact is not perceived in this way by the primary care professionals. Fewer preventive activities are carried out with immigrants, who suffer from more labour and psychosomatic problems.

Key words. Immigrants. Morbidity. Health centres. Primary health care.
1. Centro de Salud de Txantrea. Pamplona.

2. Instituto de Salud Pública de Navarra. Pamplona.

3. Centro de Salud de Azpilagaña. Pamplona.

4. Centro de Salud Milagrosa. Pamplona.

5. Centro de Salud Cascante y Buñuel. Navarra.

6. Universidad Pública de Navarra. Pamplona.

7. Centro de Salud de Berriozar. Navarra.

Recepción el 9 de marzo de 2010

Aceptación provisional el 15 de abril de 2010

Aceptación definitiva el 17 de julio de 2010

\section{Correspondencia \\ Maria Carmen Fuertes Goñi \\ Centro de Salud de Txantrea \\ San Cristóbal, s/n \\ 31015 Pamplona \\ E-mail: mfuertesg@meditex.es}

\section{Financiación}

Este proyecto ha sido financiado por el departamento de Salud del Gobierno de Navarra (85/06) y parcialmente por el CIBER de Epidemiología y Salud Pública (CIBERESP) del Instituto de Salud Pública de Navarra, proyecto "Análisis de factores condicionantes de servicios de salud y su utilización por personas inmigrantes relacionados con la promoción de salud". 


\section{INTRODUCCIÓN}

La inmigración en España ha irrumpido desde final del siglo XX de forma creciente $^{1}$ y el personal socio-sanitario requiere conocer las características de este fenómeno y disponer de conocimientos oportunos para su correcta atención ${ }^{2,3}$. El contacto inicial de las personas inmigrantes con el sistema sanitario son los servicios de atención primaria de salud (APS) y urgencias, y en estos ámbitos es necesario conocer la dimensión que tiene el fenómeno de la inmigración, los factores que influyen en su atención y los motivos por los que consultan ${ }^{4-7}$.

La población inmigrante extracomunitaria en Navarra se duplicó en cinco años hasta llegar al $10,9 \%$ en $2006^{8}$.

El presente estudio plantea como objetivos analizar la utilización de servicios en los centros de salud, comparando entre población autóctona e inmigrante, y conocer la visión sobre inmigración y salud de los profesionales de APS. Todo con la finalidad última de conocer los cambios que la inmigración ha provocado en la estructura sanitaria y en sus profesionales.

\section{MATERIAL Y MÉTODOS}

Se realizó un estudio de base poblacional, descriptivo, transversal en población atendida en APS. Complementariamente se utilizó metodología cualitativa de 6 grupos de discusión y 5 entrevistas en profundidad a profesionales relevantes y/o relacionados con la gestión de centros de salud.

\section{Población de estudio}

Se realizó una selección intencional de seis zonas básicas de salud de Navarra (San Jorge, Tudela-Este, Cintruénigo, Peralta, Milagrosa y Burlada) con mayor porcentaje de población inmigrante (PI) y, además, representativas de las diferencias económicas, demográficas y geográficas de Navarra ( 3 en zona media y 3 del sur, excluyendo la zona norte con un porcen- taje pequeño de PI). Su cuota de PI oscilaba entre el $14,50 \%$ y el $20,29 \%$ del total de población y en diciembre de 2006 tenían asignadas 86.966 personas (12.546 inmigrantes). La procedencia más frecuente de la PI era Ecuador en todas las zonas, con porcentajes cercanos al $50 \%$. En segundo lugar Colombia, salvo en Peralta que era Marruecos, que ocupaba el tercer lugar para el resto de las zonas.

En los grupos de discusión (GD) participaron 19 médicos, 10 enfermeras, 4 pediatras, 6 gestores y 2 trabajadores sociales procedentes de 14 centros de salud (1 GD con distintos profesionales de equipo de atención primaria (EAP) de la comarca de Pamplona; 2 GD, uno de médicos de familia y otro de enfermeras de la zona de Pamplona; 2 GD de la zona sur de Navarra, uno de médicos y otro de pediatras y enfermeras de pediatría; 1 grupo de directores de EAP). Los GD fueron homogéneos, respecto a población atendida, y heterogéneos en cuanto a sexo, experiencia laboral y cualificación de los profesionales. Las entrevistas en profundidad (EP) se hicieron a dos médicos, un gerente, una enfermera y una trabajadora social. Se grabaron y trascribieron los GD y EP y se hizo un análisis sociológico del discurso, triangulación entre investigadores y valoración final.

\section{Bases de datos y variables analizadas}

Se utilizó la base de datos de la tarjeta individual sanitaria (TIS) para conocer la población adscrita al final del 2006 a los centros de salud (CS) seleccionados y su país de origen. Se consideró a personas nacidas en España como "autóctonos" y en otras nacionalidades como "inmigrantes». Se consideró la accesibilidad al sistema público como universal al poder inscribirse los inmigrantes al CS a los 15 días de su llegada, independientemente de su situación administrativa regular o no. Esta accesibilidad de la PI se corroboró en los grupos de discusión.

Se obtuvieron de la base TIS las variables: "edad", "sexo", "país de origen" $y$ "fe- 
cha de alta en el sistema sanitario" para comparar comportamientos entre inmigrantes recién llegados o "nuevos" (dados de alta en TIS en el 2005 o 2006), y «asentados" (dados de alta en TIS antes del 2005).

Se utilizó la base de datos del programa informático de consultas de APS (OMI$\mathrm{AP}$ ), extrayendo variables sobre actividad durante 2006: "consultas" (con médico, enfermera o trabajador social), "derivaciones" a especialidades y "analíticas" solicitadas. Igualmente "episodios" por los que se consultó, clasificando como "episodios nuevos" atendidos en 2006 un total de 200.076. Se utilizó la clasificación CIAP-2.

Las derivaciones se analizaron para población de 15 a 44 años, por ser el tramo en el que mayores cuotas de población autóctona e inmigrante existen y, al producirse más derivaciones, la comparación de datos es más factible.

Se analizaron determinados episodios, relacionados con aparatos o sistemas del cuerpo humano, que la bibliografía considera que frecuentemente son manifestaciones de situaciones subyacentes, expresadas como somatizaciones y, por tanto, registradas como motivos de consulta orgánicos en consulta. Se definieron dos categorías: problemas relacionados con origen psicosomático y con origen laboral.

Entre los de posible origen psicosomático se incluyeron los códigos diagnósticos de: "dolor abdominal no especificado", "epigastralgia", "cefalea" y "astenia". Entre problemas de posible origen laboral se incluyeron: "lumbalgia", "cervicalgia", "dorsalgia", "gonalgia», "dolor muscular", «heridas» $\mathrm{y}$ «dermatitis de contacto/eccema".

El análisis estadístico se hizo con el programa SPSS14. Se utilizaron dos modelos de regresión logística, tomando en el primero como variable dependiente haber acudido al menos una vez al CS y en el segundo modelo haber superado la mediana de número de consultas/año (4 consultas/ año). Las variables independientes fueron: origen (autóctono o inmigrante), grupos de edad quinquenales, sexo, antigüedad y zona básica (ZB) de salud.

Para las variables cualitativas se utilizó el test chi cuadrado. Para las variables cuantitativas se utilizó la prueba t de Student de comparación de medias si la distribución era normal y pruebas no paramétricas, como el test de Mann Whitney o el test de Kolmogorov Smirnoff, cuando la distribución de las variables no se ajustaba a la normal. Se calcularon odds ratio y sus intervalos de confianza y consideraron significativas las diferencias si el IC $95 \%$ excluye el valor 1 .

\section{RESULTADOS}

Un $82 \%$ de autóctonos (61.024 sobre 74.420 con TIS al final del 2006) contactaron con el CS en el período estudiado y entre inmigrantes fueron un $72,4 \%$ [ $-0,42$ (I.C. 95\% 40-45\%)]. De 12.546 inmigrantes adscritos al CS, el 70\% tenían 25-44 años y el índice de masculinidad era 1,09. La utilización de servicios de APS por PI y autóctona y los resultados globales puede apreciarse en la tabla 1. 
Tabla 1. Utilización de servicios de atención primaria por población autóctona e inmigrante.

\begin{tabular}{|l|l|c|c|c|c|c|}
\hline & & Media & $\begin{array}{c}\text { Desv. } \\
\text { típica }\end{array}$ & Mediana & $\begin{array}{c}\text { Cuartil } \\
25\end{array}$ & $\begin{array}{c}\text { Cuartil } \\
75\end{array}$ \\
\hline \multirow{3}{*}{ Consultas/persona/año* } & Autóctonos & 6,96 & 8,83 & 4 & 1 & 10 \\
\cline { 2 - 7 } & Inmigrantes & 3,59 & 4,91 & 2 & 0 & 5 \\
\cline { 2 - 7 } & Total & $\mathbf{6 , 4 7}$ & $\mathbf{8 , 4 6}$ & $\mathbf{4}$ & $\mathbf{1}$ & $\mathbf{9}$ \\
\hline \multirow{3}{*}{ Analíticas/persona/año* } & Autóctonos & 0,37 & 0,81 & 0 & 0 & 0 \\
\cline { 2 - 8 } & Inmigrantes & 0,27 & 0,72 & 0 & 0 & 0 \\
\cline { 2 - 8 } & Total & $\mathbf{0 , 3 6}$ & $\mathbf{0 , 8}$ & $\mathbf{0}$ & $\mathbf{0}$ & $\mathbf{0}$ \\
\hline \multirow{3}{*}{ Derivaciones/persona/año* } & Autóctonos & 0,46 & 0,84 & 0 & 0 & 1 \\
\cline { 2 - 8 } & Inmigrantes & 0,32 & 0,7 & 0 & 0 & 1 \\
\cline { 2 - 7 } & Total & $\mathbf{0 , 4 4}$ & $\mathbf{0 , 8 3}$ & $\mathbf{0}$ & $\mathbf{0}$ & $\mathbf{1}$ \\
\hline
\end{tabular}

*El análisis de las diferencias encontradas entre autóctonos e inmigrantes, desagregadas por grupos de edad y sexo, eran significativas para todos los grupos $(\mathrm{p}<0,001)$.

Por edades encontramos menor probabilidad de frecuentación para inmigrantes en todos los grupos etáreos excepto entre 15-24 años (Tabla 2).

Tabla 2. Probabilidad de consultar, en el centro de salud, de inmigrantes respecto a la población autóctona.

\begin{tabular}{|l|c|c|c|c|}
\hline & \multicolumn{2}{|c|}{ Al menos una visita } & \multicolumn{2}{c|}{ Cuatro o más visitas } \\
\hline Edad & OR & IC 95\% & OR & IC 95\% \\
\hline Todas las edades & 0,80 & $0,76-0,84$ & 0,88 & $0,84-0,92$ \\
\hline Infantil (0-15años) & 0,64 & $0,54-0,76$ & 0,59 & $0,52-0,66$ \\
\hline $15-24$ & 0,88 & $0,77-1^{*}$ & 1,06 & $0,94-1,2^{*}$ \\
\hline $25-44$ & 0,87 & $0,81-0,92$ & 1,02 & $0,96-1,09^{*}$ \\
\hline $45-64$ & 0,70 & $0,62-0,80$ & 0,76 & $0,68-0,86$ \\
\hline
\end{tabular}

OR ajustados por sexo, edad, antigüedad en zona básica y pertenencia a centro de salud. *A excepción de los grupos señalados, todos los demás presentan diferencias significativas.

Entre 25-44 años, considerando su antigüedad en el sistema sanitario, no encontramos diferencias significativas en número de consultas en el caso de las mujeres «nuevas" pero sí entre hombres $(3,16 / 2,57$; $\mathrm{p}<0,000$ ), superior para los autóctonos $\mathrm{y}$ también ocurre en el caso de los hombres «asentados» $(3,20 / 2,66 ; \mathrm{p}<0,000)$. Las mujeres inmigrantes que llevan más de dos años ("asentadas") superan las consultas de las autóctonas $(4,63 / 4,19 ; \mathrm{p}<0,000)$.

Sin embargo, los profesionales perciben "sobrecarga» con la irrupción de la PI en la consulta. En los GD y EP destacan algunos factores e imágenes que sustentan esta autopercepción:

- Desconocer los antecedentes de la PI añade incertidumbre a la relación asistencial y condiciona la dinámica de consulta, incorporando duda y sospecha sobre patología previa.

- La movilidad residencial de la PI.

- La multiculturalidad o encontrar personas con diferentes hábitos, costumbres, prácticas y códigos lingüísticos. 
- El distinto concepto del tiempo, identificándose impuntualidad o inasistencia a las citas, sobre todo entre la población latinoamericana.

Globalmente se percibe desorden en la consulta, como refleja un comentario de uno de los grupos de discusión:

"..es cierto que llegan tarde el $90 \%$ o que no vienen, cosa que habría que valorar; si no vienen por su idiosincrasia o porque realmente están en trabajos que no les dejan salir, que también podría ser...» (GD6)

\section{Consultas}

El porcentaje de PI con al menos una consulta al CS es menor en inmigrantes para todos los grupos de edad (IC 95\% 1624\%) (Fig. 1).

Figura 1. Distribución por grupos de edad de los autóctonos e inmigrantes que consultaron al menos una vez en 2006 a atención primaria.

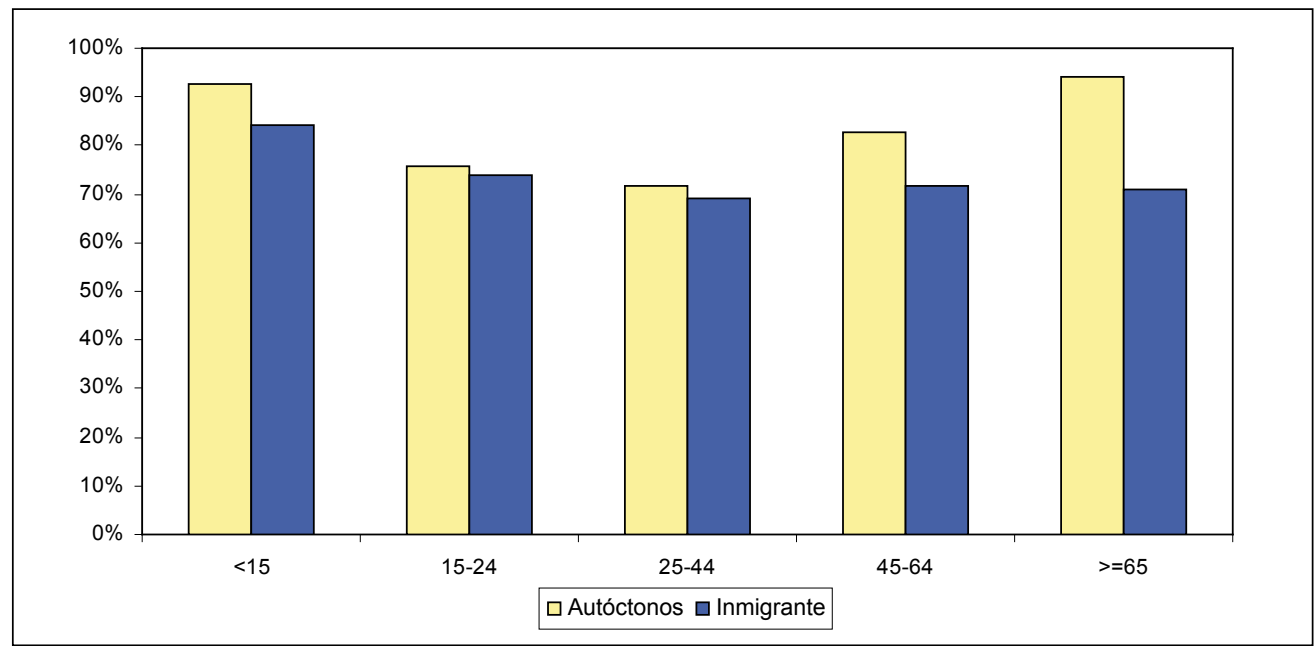

Las diferencias son significativas $(\mathrm{p}<0,01)$ en todos los grupos de edad excepto en el del 15-24 años.

Al diferenciar consultas según profesional, este menor porcentaje de inmigrantes se mantiene para consultas con médico y enfermera, pero con la trabajadora social el porcentaje es mayor en inmigrantes, tanto asentados como nuevos, aunque con la edad se van igualando con los autóctonos.

La probabilidad comparada de tener al menos 1 ó 4 y más consultas/año en el CS es menor en inmigrantes respecto autóctonos, como puede apreciarse en la tabla 2. Las diferencias son significativas sobre todo en el caso de la población infantil.

Según análisis multivariante, la probabilidad de tener al menos una consulta al año y también de tener 4 ó más consultas/ año, es mayor en las mujeres respecto a los hombres, también en menores de 5 años y mayores de 64 años respecto a los otros grupos de edad. Las personas con alta más reciente en la ZB (últimos dos años) tenían más probabilidad de tener una o más visitas respecto a las "asentadas». La probabilidad de tener cuatro o más consultas sin embargo fue más alta en "asentados".

Las diferencias observadas entre autóctonos e inmigrantes son independientes de la distinta distribución en esos grupos por sexo, edad, CS y antigüedad en la ZB (Tabla 2).

Los profesionales de los CS, en los GD y EP, sin embargo perciben el problema de 
sobrecarga que ha creado la atención a la PI pero en algunos casos atribuyen el problema no tanto a la cantidad de consultas sino a la dificultad que conllevan éstas:

...la difícil relación que se establece con estas personas, desde el punto de vista cultural, desde el punto de vista idiomático, supone una pérdida de tiempo, una dificultad de interpretar lo que ellos quieren decir, con los riesgos que supone a la hora de hacer el diagnóstico y el tratamiento... (GD4).

\section{Derivaciones}

La tabla 3 recoge datos de las derivaciones en porcentajes superiores al 1\% para ambas poblaciones. Destaca la mayor derivación de PI a atención ginecoobstétrica y urgencias, mientras la autóctona fue derivada en proporción mayor al resto de especialidades y especialmente a alergología, cirugía general, salud mental, otorrinolaringología, dermatología y traumatología.

Tabla 3. Derivaciones a atención especializada en población autóctona e inmigrante de 15-44 años.

\begin{tabular}{|l|c|c|c|}
\hline & Autóctonos & Inmigrantes & Valor p \\
\hline $\mathrm{N}$ & 8.592 & 2.449 & \\
\hline Traumatología & $18 \%$ & $16 \%$ & 0,02 \\
\hline Ginecología-Obstetricia & $16 \%$ & $26 \%$ & $<0,001$ \\
\hline Dermatología & $15 \%$ & $12 \%$ & $<0,001$ \\
\hline Oftalmología & $10 \%$ & $11 \%$ & 0,16 \\
\hline Urgencias & $4 \%$ & $7 \%$ & $<0,001$ \\
\hline Digestivo & $5 \%$ & $6 \%$ & 0,05 \\
\hline Otorrinolaringología (ORL) & $8 \%$ & $6 \%$ & 0,001 \\
\hline Salud mental & $6 \%$ & $3 \%$ & $<0,001$ \\
\hline Cirugía general & $5 \%$ & $4 \%$ & 0,04 \\
\hline Neurología & $3 \%$ & $3 \%$ & 0,96 \\
\hline Urología & $3 \%$ & $3 \%$ & 0,96 \\
\hline Alergología & $4 \%$ & $1 \%$ & $<0,001$ \\
\hline
\end{tabular}

\section{Episodios nuevos}

El más frecuente fue la infección respiratoria alta (CIAP: R74), superior al 7\% del total de episodios, en todos los grupos de edad (entre $7,8 \%$ en inmigrantes de 45 a 64 años y $23,6 \%$ en autóctonos menores de 15 años). El $2^{\circ}$ episodio más frecuente se comporta diferente en cada grupo de edad. Así, en menores de 15 años corresponde al episodio de "fiebre» en autóctonos (4,6\%), mientras que en inmigrantes es "tos" (3,5\%). En el grupo de 15 a 24 años es «amigdalitis aguda" para autóctonos $(3,4 \%)$ y en inmigrantes "problemas acceso sistema sanitario" $(5,1 \%)$, que ocupa también el $2^{\text {o }}$ lugar entre $25-44(3,7 \%)$ y entre 45 a 64 años $(3,2 \%)$. Este episodio no aparece en autóctonos. La «lumbalgia» es motivo de consulta en $2^{\circ}$ ó $3^{\text {er }}$ lugar en mayores de 25 años, tanto en autóctonos como inmigrantes.

Entre el 60\% de episodios más frecuentes por edades, aparecen únicamente en autóctonos y no en inmigrantes: en menores de 15 años "actividades preventivas", en 15-44 años "tabaquismo" y en 45-64 años sí aparece "tabaquismo" en inmigrantes pero con menor frecuencia $(0,7 \%$ vs. $1 \%)$.

$\mathrm{Al}$ agrupar episodios nuevos según la clasificación internacional de AP (CIAP-2), (Tabla 4), los grupos con mayor porcen- 
taje de consultas entre inmigrantes son: "problemas sociales", en todos las edades, (en hombres 4 veces más frecuentes que en autóctonos); "sistema nervioso" y "aparato digestivo" y en mujeres el de los "gineco-obstétricos». En autóctonos son más frecuentes "problemas respiratorios", "psicológicos", "problemas auditivos", "circulatorios" $\mathrm{y}$ "problemas generales e inespecíficos» $(\mathrm{p}<0,001)$.

Tabla 4. Consultas de autóctonos e inmigrantes por episodios nuevos durante 2006, distribuidos por grupos CIAP.

\begin{tabular}{|c|c|c|c|c|c|c|c|c|}
\hline \multirow[b]{2}{*}{$\mathbf{N}$} & \multicolumn{4}{|c|}{ Mujeres } & \multicolumn{4}{|c|}{ Hombres } \\
\hline & 112.138 & 97.935 & 14.203 & $\mathbf{p}$ & 87.938 & 77.284 & 10.654 & $\mathbf{p}$ \\
\hline Código CIAP & Total & Autóct. & Inmig. & & Total & Autóct. & Inmig. & \\
\hline Problemas generales e inespecíficos & $8,0 \%$ & $8,2 \%$ & $6,5 \%$ & $<0,001$ & $8,9 \%$ & $9,1 \%$ & $7,9 \%$ & $<0,001$ \\
\hline Sangre, órganos hematop. y sist. inmunitario & $1,1 \%$ & $1,0 \%$ & $1,4 \%$ & $<0,001$ & $0,7 \%$ & $0,8 \%$ & $0,6 \%$ & 0,03 \\
\hline Aparato digestivo & $10,9 \%$ & $10,7 \%$ & $12,0 \%$ & $<0,001$ & $12,0 \%$ & $11,8 \%$ & $13,4 \%$ & $<0,001$ \\
\hline Ojo y anejos & $4,4 \%$ & $4,4 \%$ & $4,2 \%$ & 0,29 & $4,8 \%$ & $4,7 \%$ & $5,4 \%$ & 0,002 \\
\hline Aparato auditivo & $4,9 \%$ & $5,2 \%$ & $2,8 \%$ & $<0,001$ & $5,3 \%$ & $5,5 \%$ & $3,5 \%$ & $<0,001$ \\
\hline Aparato circulatorio & $3,3 \%$ & $3,4 \%$ & $2,5 \%$ & $<0,001$ & $3,0 \%$ & $3,2 \%$ & $1,7 \%$ & $<0,001$ \\
\hline Aparato locomotor & $12,5 \%$ & $12,5 \%$ & $12,4 \%$ & 0,73 & $12,1 \%$ & $11,7 \%$ & $14,8 \%$ & $<0,001$ \\
\hline Sistema nervioso & $2,9 \%$ & $2,8 \%$ & $3,9 \%$ & $<0,001$ & $2,2 \%$ & $2,1 \%$ & $3,2 \%$ & $<0,001$ \\
\hline Problemas psicológicos & $3,3 \%$ & $3,4 \%$ & $2,6 \%$ & $<0,001$ & $3,2 \%$ & $3,3 \%$ & $2,5 \%$ & $<0,001$ \\
\hline Aparato respiratorio & $24,0 \%$ & $24,8 \%$ & $18,5 \%$ & $<0,001$ & $27,3 \%$ & $28,0 \%$ & $22,0 \%$ & $<0,001$ \\
\hline Piel y faneras & $11,3 \%$ & $11,4 \%$ & $10,7 \%$ & 0,01 & $12,2 \%$ & $12,1 \%$ & $13,3 \%$ & $<0,001$ \\
\hline Aparato endocrino, metabolismo y nutrición & $2,8 \%$ & $2,8 \%$ & $2,6 \%$ & 0,17 & $2,5 \%$ & $2,6 \%$ & $2,1 \%$ & 0,002 \\
\hline Aparato urinario & $3,3 \%$ & $3,2 \%$ & $3,5 \%$ & 0,06 & $1,7 \%$ & $1,7 \%$ & $1,5 \%$ & 0,14 \\
\hline Planific. familiar, embarazo, parto y puerperio & $1,8 \%$ & $1,4 \%$ & $4,6 \%$ & $<0,001$ & $0,0 \%$ & $0,0 \%$ & $0,0 \%$ & $\begin{array}{c}\text { No } \\
\text { procede }\end{array}$ \\
\hline Aparato genital femenino y mamas & $3,4 \%$ & $3,0 \%$ & $6,1 \%$ & $<0,001$ & $0,0 \%$ & $0,0 \%$ & $0,0 \%$ & $\begin{array}{c}\text { No } \\
\text { procede }\end{array}$ \\
\hline Aparato genital masculino & $0,0 \%$ & $0,0 \%$ & $0,0 \%$ & $\begin{array}{c}\text { No } \\
\text { procede }\end{array}$ & $1,8 \%$ & $1,8 \%$ & $1,7 \%$ & 0,47 \\
\hline Problemas sociales & $2,2 \%$ & $1,7 \%$ & $5,6 \%$ & $<0,001$ & $2,1 \%$ & $1,5 \%$ & $6,3 \%$ & $<0,001$ \\
\hline
\end{tabular}

La tabla 5 refleja episodios indicativos de posible patología laboral y patología psicosomática entre 25-44 años, que concentra sobre todo población activa. 
Tabla 5. "Problemas psicosomáticos» y de "posible origen laboral» por los que consultan por primera vez en 2006 la población autóctona e inmigrante.

\begin{tabular}{|c|c|c|c|c|c|c|c|c|}
\hline & \multicolumn{4}{|c|}{ Mujeres } & \multicolumn{4}{c|}{ Hombres } \\
\hline & Autóctonas & Inmigrantes & Total & Valor p & Autóctonos & Inmigrantes & Total & Valor p \\
\hline $\mathrm{N}$ & 23.124 & 7.692 & 30.816 & & 17.155 & 5.565 & 22.720 & \\
\hline $\begin{array}{c}\text { Problemas } \\
\text { psicosomáticos }\end{array}$ & $2,3 \%$ & $4,4 \%$ & $2,8 \%$ & $\mathrm{P}<0,001$ & $1,8 \%$ & $4,1 \%$ & $2,4 \%$ & $\mathrm{P}<0,001$ \\
\hline $\begin{array}{c}\text { Posible origen } \\
\text { laboral }\end{array}$ & $6,1 \%$ & $7,7 \%$ & $6,5 \%$ & $\mathrm{P}<0,001$ & $8,2 \%$ & $11,3 \%$ & $9,0 \%$ & $\mathrm{P}<0,001$ \\
\hline
\end{tabular}

Problemas posiblemente psicosomáticos: dolor abdominal no especificado, epigastralgia, cefalea y astenia.

Problemas de posible origen laboral: lumbalgia, cervicalgia, dorsalgia, gonalgia, dolor muscular, herida y dermatitis de contacto/eccema.

Los indicativos de patología psicosomática son más frecuentes en inmigrantes para todos los grupos de edad, tanto en hombres como en mujeres.

Los indicativos de posible patología laboral son más frecuentes en inmigrantes en todos los grupos de edad excepto entre 45 y 64 años.

Los profesionales sanitarios también perciben que la PI tiene similar patología que la autóctona, salvo excepciones como heridas de arma blanca, enfermedades parasitarias como malaria o más incidencia de tuberculosis. No obstante, en los GD y EP se constata una distinta vivencia de la enfermedad por parte de la PI para quienes la prioridad no es la salud, sino el trabajo, lo que puede conllevar más demandas agudas e intervenciones puntuales en PI. Se describen en esta población muchas somatizaciones vinculadas al proceso de adaptación y desarraigo, procesos a veces de patología difusa y tendencia, especialmente los latinoamericanos, al relato, dando muchos rodeos sobre lo que les pasa, sin definir claramente una patología concreta.

En los GD y EP se incide en la dificultad de los aspectos relacionales con la PI, en la dimensión emocional del encuentro sanitario y se concluye que influye mucho la actitud de los profesionales.

«... estoy convencido que el hecho de ser inmigrante influye en la forma de relación de los profesionales con los pacientes, en unos casos para un mayor paternalismo y en otros para una relación más distante...» EP5.

“... reflexionar sobre la diferencia en las consultas a los niños inmigrantes te da la sensación como de vivir en la España de hace muchos años. EP4.

«... en niños encontramos más problemas de parásitos cuando llegan...»GD1

"...tienen los peores trabajos, entonces tienen una patología laboral gorda... hay muchas lumbalgias, mucho problema osteoarticular, mucha depresión, las mujeres estân todas deprimidas, a su vez no llevan tratamiento antidepresivo, tú les planteas ponerles y no les entra...»GD4.

Por tanto se confirma lo encontrado en el estudio cuantitativo respecto a los motivos de consulta de la PI.

Además, la inmigración irrumpe en un contexto de crisis de la atención primaria con mayor tecnificación de la gestión; se percibe como una sobrecarga y amplifica una realidad previa de necesidad de cambio. También se perciben aportaciones del fenómeno migratorio en sus aspectos positivos $\mathrm{y}$, por tanto, como oportunidad de mejora. Se remarca que deja más palpable la necesidad de una atención integral a la población y de la importancia de lo socio-sanitario y de la educación y promoción de la salud.

«...los valores de lo comunitario vuelven a crecer, pero yo sí creo que el tener que abordar el fenómeno de la inmigración puede ser una oportunidad...»EP5 
Los profesionales de los centros de salud sienten aislamiento en lo relativo a la atención a la PI. El fenómeno migratorio se percibe de forma diferente entre profesionales de enfermería, trabajo social y medicina. Los médicos reclaman mejor distribución de las cargas de trabajo y mayor implicación de enfermería en la consulta de acogida del paciente inmigrante. Trabajo social aumenta su protagonismo. Los colectivos de inmigrantes no son percibidos como un todo homogéneo sino diversos en función del origen, tiempo en la nueva sociedad, carácter regular o irregular o cercanía al modelo cultural del profesional.

Otras demandas constatadas en los GD y EP eran:

- No procede crear estructuras, servicios o procedimientos específicos para inmigrantes, pero sí materiales de ayuda como folletos informativos en distintos idiomas, etc.

- Formación en multiculturalidad para los profesionales.

- Valorar alternativas ajustadas a las condiciones laborales de la PI para el cuidado de problemas crónicos.

- Profundizar en detectar problemas psicosomáticos o laborales para adecuar soluciones.

- Necesidad de abordajes integrales e intersectoriales entre las distintas administraciones. Puntualmente se requieren "traductores", mediadores culturales", o "agentes de salud" de las diferentes comunidades más predominantes de PI, para influir en hábitos saludables desde sus valores culturales y medicina tradicional.

\section{DISCUSIÓN}

La probabilidad de tener al menos una visita/año, y también la de al menos 4 visitas al año, es mayor para población autóctona que para inmigrante de forma significativa, independientemente de la edad, la zona o el tiempo de residencia. Esto coincide con otros estudios ${ }^{9,10}$ que argumentan la diferencia es debida en parte al mejor estado de salud de los inmigrantes, aunque también habría que valorar si hay un mayor umbral para buscar asistencia sanitaria. Esta menor frecuentación de servicios por inmigrantes contrasta con las percepciones contrarias de profesionales, que puede atribuirse a que las consultas con inmigrantes requieren más tiempo y esfuerzo por parte del profesional, tal como se constata en el estudio cualitativo.

La comparación de utilización de servicios de inmigrantes, considerando su antigüedad en el sistema sanitario, se limitó a la población de 25-44 años, ya que es el grupo de edad más numeroso de PI (22,7\% de la población total de esa edad). Las mujeres inmigrantes que llevan más de dos años ("asentadas") superan las consultas de las autóctonas, probablemente por causas ginecoobstétricas. Entre los hombres, los autóctonos tienen mayor número de consultas, tanto los «asentados» como los "nuevos".

La PI precisa de derivación a atención urgente en mayor proporción que la autóctona (Tabla 3), por lo que la frecuentación de la PI en servicios hospitalarios de urgencias sería algo a considerar en próximos estudios. No pudo recogerse por separado la atención en horario habitual de los CS y la atención continuada o de servicios de urgencias extrahospitalarios. En los dos centros de Pamplona, las consultas que realizaban por la tarde o noche los inmigrantes no estaban registradas. Este análisis permitiría valorar en conjunto si la menor frecuentación de los inmigrantes a APS es debida a su mejor estado de salud o a mayor uso de servicios de urgencias en vez de CS.

En derivaciones a otras especialidades, hay un porcentaje superior en autóctonos hacia servicios de traumatología, dermatología, otorrinolaringología, salud mental, cirugía general y alergología, que las de PI. Esto puede estar condicionado porque la $\mathrm{PI}$, tal como constatan otros estudios, son sobre todo jóvenes, sanos y fuertes ${ }^{11}$. La excepción es en cuanto a la derivación a urgencias, que pudiera indicar que los inmigrantes cuando acuden al centro de salud es con problemas importantes. 
Las mujeres inmigrantes "asentadas", entre 25-44 años, tienen un número ligeramente mayor de consultas y analíticas que las autóctonas. Quizás haya una progresiva acomodación de los comportamientos de uso de la PI a los de la población autóctona, o bien al estar "asentadas" las inmigrantes empiezan a preocuparse de los problemas preventivos ginecológicos o de embarazo y acuden a APS para consultar por estos, mientras que las autóctonas van directamente a los centros de atención a la mujer. Esto puede indicarnos, al igual que otros estudios ${ }^{12}$, una necesidad de facilitar información en utilización de servicios a PI sobre la que hay experiencia.

Aunque el número de consultas es menor en inmigrantes, el total de episodios nuevos por los que consultan, sobre todo los hombres inmigrantes y dados de alta en TIS en 2005-2006 ("nuevos»), es mayor que en los autóctonos, probablemente por tener una primera consulta con el trabajador social de alta en TIS y acceso al sistema sanitario, episodio que no aparece los autóctonos. Otras posibles explicaciones serían que los inmigrantes acuden menos al CS y al hacerlo aprovechan esa visita para consultar por más episodios diferentes en esa visita, o que los autóctonos consultan frecuentemente por episodios crónicos no contabilizados como nuevos.

Sobre los episodios por los que se consulta por primera vez, podemos considerar que, si un $81 \%$ de la población ha contactado con APS y el sistema público tiene cobertura universal, los datos obtenidos se aproximan mucho a la morbilidad en incidencia de enfermedades o problemas, ya que se han analizado los episodios abiertos como «nuevos» en 2006.

La frecuencia notablemente más elevada en inmigrantes de episodios indicativos de posibles problemas psicosomáticos, puede indicar que los problemas de salud mental serían más numerosos y mayor la diferencia con autóctonos, especialmente si consideramos entre éstos los episodios que pueden indicar somatización. Es importante que la formación a los profesio- nales sanitarios en el tema de inmigración, como se demanda en los GD y en EP, tenga en cuenta estos aspectos, así como la atención al duelo de las personas inmigrantes ${ }^{13}$. Según el psiquiatra García Campayo ${ }^{14}$, la adaptación a la atención del paciente inmigrante viene dificultada porque en la medicina occidental se tiende a diferenciar entre síntomas físicos y psicológicos y al desarrollo de categorías diagnósticas como los trastornos somatomorfos, un trastorno difícil de resolver en nuestro sistema médico; mientras que en otras medicinas el abordaje es más holístico ${ }^{15}$.

Se ha encontrado que los problemas asociados a posible origen laboral son más frecuentes en inmigrantes, al igual que en otro estudio realizado en Navarra ${ }^{16}$ que concluye que los accidentes laborales son más frecuentes y graves en PI. Estudios recientes destacan la influencia de las condiciones laborales en la salud de inmigrantes ${ }^{17}$.

A modo de conclusiones, se puede deducir que:

- La población autóctona utiliza más los servicios sanitarios que la inmigrante tanto en el CS como en derivaciones a atención especializada, aunque los profesionales sanitarios perciben lo contrario.

- Entre las dificultades en la atención a inmigrantes se apunta la falta de formación de los profesionales en competencia cultural que no se imparte en el pregrado ni en el postgrado de las carreras sanitarias. Las dificultades idiomáticas hacen las entrevistas clínicas más dificultosas y largas ${ }^{18}$. Una mejor atención a la PI requeriría un esfuerzo de los organismos de formación para sanitarios ${ }^{19-21}$ y de la Administración para facilitar la mediación cultural que puede requerirse en algunos $\operatorname{casos}^{22,23}$.

- Se aprecia que la PI requiere en mucha mayor proporción servicios de atención gineco-obstétrica, coincidiendo con otros estudios ${ }^{24}$ y con mayor natalidad en inmigrantes ${ }^{25}$. 
- La patología que el estudio ha considerado como indicativa de posibles problemas psicosomáticos y la de problemas laborales presenta una frecuencia notablemente más elevada en inmigrantes.

- Respecto a actividades preventivas, se detecta que no se realizan de manera habitual con la PI. La atención más frecuente prestada a la PI en el CS se orienta, sobre todo, a problemas administrativos y patología aguda y sería adecuado plantear la realización con la PI de algunas actividades preventivas.

Como limitaciones del estudio, destacamos que puede existir un sesgo por la mayor movilidad geográfica de los inmigrantes. Podrían no coincidir exactamente los contabilizados en la TIS al final del año de estudio con quienes utilizaron los servicios durante ese año. Igualmente puede haber inmigrantes registrados en los centros de salud estudiados a final de 2006 que sólo hayan estado una parte de ese año atendidos en los centros estudiados. Se estima que los datos son próximos a la realidad y las variaciones censales también se producen, aunque en menor medida, en la población autóctona. En 2006 se considera que la TIS en Navarra era muy accesible a todos los inmigrantes y su actualización era ágil, por lo que se puede estimar que los errores en las bases de datos son mínimos.

Los episodios nuevos se estudiaron de forma retrospectiva y se registraron según códigos de la clasificación CIAP-2, que no todos los profesionales utilizan de la misma manera. Puede haber variabilidades interindividuales de registro para procesos similares. Se constata la limitación de los códigos de la CIAP-2, orientados más a registros de problemas bio-médicos que psicosociales, que son de capital importancia en la PI. Así, los "problemas administrativos" que aparecen entre las principales causas de consulta, agrupan variados tipos de demandas y problemas sobre los que se interviene, más allá de resolver problemas de accesibilidad al sistema sanitario.

\section{Agradecimientos}

Queremos agradecer a Javier Apezteguía y a la Dirección de Atención Primaria que nos ha facilitado el acceso a las bases de datos. También nuestro agradecimiento para la Sección de Promoción de Salud del Instituto de Salud Pública que nos ha animado a la realización de este trabajo y a Nerea Egüés y Marcela Guevara, que han ayudado en el tratamiento estadístico de los datos. Igualmente nuestro agradecimiento a Antonio Brugos por las sugerencias para la preparación del presente artículo y a todos los profesionales de APS que han participado en los grupos de discusión y entrevistas en profundidad.

\section{BIBLIOGRAFÍA}

1. Encuesta de migraciones. Serie 2006-2008. INE. 2009. Disponible en http://www.ine. es $/$ jaxi $/$ menu.do?type $=$ pcaxis $\&$ path $=/$ t $20 /$ p311/2006-2008\&file $=$ pcaxis

2. López Abuin JM. El médico de familia ante el abordaje sociosanitario del inmigrante. FMC. Form Med Contin Aten Prim 2002; 9: 492-497.

3. Pertínez J, Clusa T. Inmigración y Atención Sanitaria. Humanitas $n^{\circ} 34$ dic 2008. Disponible en: http://www.fundacionmhm.org/revista. html

4. Martín M. En: Guía de Atención al inmigrante. Sociedad Madrileña de Medicina Familiar y Comunitaria Madrid: Ergon 2003.

5. Seguí M. El inmigrante en la consulta del médico de cabecera. Semergen 2005; 31: 505507.

6. Pena M. Motivos de consulta y características demográficas de una comunidad de inmigrantes "sin papeles" en el distrito de UseraVillaverde (Madrid). Aten Primaria 2001; 27: 25-28.

7. Cots F, Castells X, García, O, Riu M, Felipe A VALL O. Impact of immigration on the cost of emergency visits in Barcelona (Spain). BMC Health Serv Res 2007; 7: 9.

8. Informe variaciones residenciales en Navarra 2006. En: http://www.cfnavarra.es/estadistica/

9. CAULFORD P, VALI Y. Providing health care to medically uninsured inmigrants and refugees. Can Med Assoc J 2006; 174: 1253-1254.

10. Carrasco-Garrido P, De Miguel AG, Barrera VH, JimÉnEz-GARCía R. Health profiles, lifestyles 
and use of health resources by the immigrant population resident in Spain. Eur J Public Health 2007; 17: 503-507.

11. Jansá JM, García de Olalla P. Salud e inmigración: nuevas realidades y nuevos retos. Gac Sanit 2004; 18 (Supl. 1): 207-213.

12. FUERTES C, MARTín MA. El inmigrante en la consulta de atención primaria. An Sist Sanit Navar 2006; 29 (Supl. 1): 9S-25S.

13. Аснотеgu J. Los duelos de la migración: una perspectiva psicopatológica y psicosocial. En: Medicina y cultura. E Perdiguero y JM Comelles (comp). Editorial Bellaterra. Barcelona 2000: 88-100.

14. García Campayo J. Usted no tiene nada. Los trastornos somatomorfos. Barcelona: Océano 1999.

15. García Campayo J, Sanz Carrillo C. La salud mental en los inmigrantes: el nuevo desafío. Med Clin (Barc) 2002; 118: 187-191.

16. Parra A, Fernández Baraibar J, García López V, AyestaRÁn JR. Extramiana E. Mercado de trabajo, salud laboral e inmigración. An Sist Sanit Navar 2006; 29 (Supl. 1): 77S-95S.

17. García AM, López-Jacob MJ, Alonso A, Ruiz-Frutos C, Ahonen EQ, Porthé V. Condiciones de trabajo y salud en inmigrantes: entrevistas a informantes clave. Gac Sanit 2009; 23: 9197.

18. DuBard CA, Gizlice Z. Language spoken and differences in health status, access to care, and receipt of preventive services among US Hispanics. Am J Public Health 2008; 98: 2021-2028.

19. Recomendaciones para la atención al paciente inmigrante en la consulta de Atención Primaria. Barcelona: Sociedad Española de Medicina Familiar y Comunitaria (semFYC): semFYC 2001. Disponible en URL http:// www.semfyc.es

20. Manual de atención sanitaria a inmigrantes $2^{\mathrm{a}}$ edición. Fundación Progreso y Salud. Consejería de Salud Junta de Andalucía. Sevilla 2004.

21. Vázquez ML, Terraza R, Vargas L, Lizana T. Necesidades de los profesionales de salud en la atención a la población inmigrante. Gac Sanit 2009; 23: 396-402.

22. Martincano JL. Valoración cultural del inmigrante. Guía de Medicina Transcultural. Madrid: IM\&C S.A; 2003:75-128.

23. Alonso E. El médico de familia ante el fenómeno migratorio. Salud e inmigración. Estrategias y recursos en Cataluña. FMC. Form Med Contin Aten Prim 2004; 11 (Supl. 3): 5.

24. Soler-González J, Serna C, Rué M, Bosch A, Ruiz MC, GerVilla J. Utilización de recursos de atención primaria por parte de inmigrantes y autóctonos que han contactado con los servicios asistenciales de la ciudad de Lleida. Aten Primaria 2008; 40: 225-233.

25. Instituto Nacional de Estadística (INE). España en cifras 2006. Disponible en: http://www. inw.es/prodyser/pubweb/espcif/pobl06.pdf 